\title{
Lorentz invariance relations for twist-3 functions and frame-independence of twist-3 cross sections
}

\author{
Yuji Koike* \\ Department of Physics, Niigata University, Ikarashi, Niigata 950-2181, Japan \\ E-mail: koikeent.sc.niigata-u.ac.jp
}

Koichi Kanazawa

Department of Physics, SERC, Temple University, Philadelphia, PA 19122, USA

E-mail: tuf29138@temple.edu

\section{Andreas Metz}

Department of Physics, SERC, Temple University, Philadelphia, PA 19122, USA

E-mail: metza@temple.edu

\section{Daniel Pitonyak}

RIKEN BNL Research Center, Brookhaven National Lab, Upton, New York 11973, USA

E-mail: dpitonyakequark.phy.bnl.gov

\section{Marc Schlegel}

Institute for Theoretical Physics, Universität Tübingen, Auf der Morgenstelle 14, D-72076

Tübingen, Germany

E-mail: marc.schlegeleuni-tuebingen.de

\begin{abstract}
In order to derive twist-3 cross sections in the collinear factorization, it is convenient to introduce three types (i.e., intrinsic, kinematical and dynamical) of twist-3 distribution and fragmentation functions. We derive a complete set of the constraint relations among those twist- 3 functions, using the QCD equation-of-motion (EOM) and the Lorentz invariance properties of the correlation functions. The twist- 3 cross sections, in general, depend on the auxiliary light-like vectors which are introduced to define the twist- 3 functions from the correlation functions. We argue that the twist-3 cross sections acquire a frame-independent (Lorentz invariant) form thanks to those constraint relations.
\end{abstract}

XXIV International Workshop on Deep-Inelastic Scattering and Related Subjects

11-15 April, 2016

DESY Hamburg, Germany

\footnotetext{
* Speaker.
} 


\section{Collinear twist-3 distribution and fragmentation functions}

We first summarize the distribution and fragmentation functions which are necessary for the calculation of twist-3 cross sections. The collinear quark distribution functions in the nucleon are defined from the lightcone correlator

$$
\Phi_{i j}(x)=\int_{-\infty}^{\infty} \frac{d \lambda}{2 \pi} \mathrm{e}^{i \lambda x}\left\langle P S\left|\bar{q}_{j}(0)[0 ; \lambda n] q_{i}(\lambda n)\right| P S\right\rangle,
$$

where $q_{i}$ is a quark field with the spinor index $i$, and $|P S\rangle$ is the nucleon state with momentum $P\left(P^{2}=M_{N}^{2}\right)$ and the spin vector $S(P \cdot S=0)$. For $P^{\mu}=(E, \vec{P})$ we define two lightlike vectors $p^{\mu}$ and $n^{\mu}$ as $p^{\mu}=\left(P^{+} / \sqrt{2}\right)(1, \vec{P} /|\vec{P}|)$ and $n^{\mu}=(1,-\vec{P} /|\vec{P}|) /\left(\sqrt{2} P^{+}\right)$with $P^{+}=(E+|\vec{P}|) / \sqrt{2}$, which satisfy $P^{\mu}=p^{\mu}+\left(M_{N}^{2} / 2\right) n^{\mu}$ and $p \cdot n=1$. Decomposition of $\Phi(x)$ defines the quark distributions listed in Table $1 .[0 ; \lambda n]$ is a gauge link connecting 0 and $\lambda n$. Likewise collinear quark fragmentation functions can be defined from the fragmentation correlator

$$
\Delta_{i j}(z)=\frac{1}{N_{c}} \sum_{X} \int_{-\infty}^{\infty} \frac{d \lambda}{2 \pi} \mathrm{e}^{-i \frac{\lambda}{z}}\left\langle 0\left|[ \pm \infty m ; 0] q_{i}(0)\right| P_{h} S_{h} ; X\right\rangle\left\langle P_{h} S_{h} ; X\left|\bar{q}_{j}(\lambda m)[\lambda m ; \pm \infty m]\right| 0\right\rangle .
$$

The hadron momentum $P_{h}\left(P_{h}^{2}=M_{h}^{2}\right)$ can also be decomposed as $P_{h}^{\mu}=p_{h}^{\mu}+\left(M_{h}^{2} / 2\right) m^{\mu}$ by introducing two lightlike vectors $p_{h}^{\mu}$ and $m^{\mu}$ which are defined similarly to $p^{\mu}$ and $n^{\mu}$. Fragmentation functions defined from $\Delta(z)$ are summarized in Table 1 . For precise definition of each distribution and fragmentation function, we refer the readers to [1]. We name the twist-3 functions defined from $\Phi(x)$ and $\Delta(z)$ as intrinsic twist-3 distribution/fragmentation functions.

\begin{tabular}{|c|c|c|c|}
\hline $\mathrm{N} \backslash \mathrm{q}$ & Ave. & $S_{\|}$ & $S_{\perp}$ \\
\hline Ave. & $f_{1}(x), e(x)$ & & \\
\hline$S_{\|}$ & & $g_{1}(x)$ & $h_{L}(x)$ \\
\hline$S_{\perp}$ & & $g_{T}(x)$ & $h_{1}(x)$ \\
\hline
\end{tabular}

\begin{tabular}{|c|c|c|c|}
\hline $\mathrm{h} \backslash \mathrm{q}$ & Ave. & $S_{\|}$ & $S_{\perp}$ \\
\hline Ave. & $D_{1}(z), E(z), H(z)$ & & \\
\hline$S_{\|}$ & $E_{L}(z)$ & $G_{1}(z)$ & $H_{L}(z)$ \\
\hline$S_{\perp}$ & $D_{T}(z)$ & $G_{T}(z)$ & $H_{1}(z)$ \\
\hline
\end{tabular}

Table 1: Collinear quark distribution functions in the nucleon (left) and quark fragmentation functions for spin-1/2 hadron (right) which are classified by the spin of the hadron and a quark. In the left table $e, h_{L}$ and $g_{T}$ are intrinsic twist-3 distributions while others are twist-2 distributions. In the right table, $E, H_{L}$ and $G_{T}$ are naively $T$-even intrinsic twist-3 functions, while $H, E_{L}$ and $D_{T}$ are naively $T$-odd intrinsic twist-3 functions. Others are twist-2.

The second type of twist-3 functions are called kinematical ones and are defined from the transverse-momentum-dependent correlators as

$$
\Phi_{\partial, i j}^{\rho}(x)=\int d^{2} k_{T} k_{T}^{\rho} \Phi_{i j}\left(x, k_{T}\right), \quad \Delta_{\partial, i j}^{\rho}(z)=\int d^{2} p_{\perp} p_{\perp}^{\rho} \Delta_{i j}\left(z, p_{\perp}\right),
$$

where $\Phi\left(x, k_{T}\right)$ and $\Delta\left(z, p_{\perp}\right)$ are given as

$$
\begin{aligned}
\Phi_{i j}\left(x, k_{T}\right)= & \int_{-\infty}^{\infty} \frac{d \lambda}{2 \pi} \int \frac{d^{2} z_{T}}{(2 \pi)^{2}} \mathrm{e}^{i x \lambda+i k_{T} \cdot z_{T}}\langle P, S| \bar{q}_{j}(0)[0 ; \infty n]\left[\infty n ; \infty n+z_{T}\right] \\
& \times\left[\infty n+z_{T} ; \lambda n+z_{T}\right] q_{i}\left(\lambda n+z_{T}\right)|P, S\rangle, \\
\Delta_{i j}\left(z, p_{\perp}\right)= & \frac{1}{N_{c}} \sum_{X} \int_{-\infty}^{\infty} \frac{d \lambda}{2 \pi} \int \frac{d^{2} z_{\perp}}{(2 \pi)^{2}} \mathrm{e}^{-i \frac{\lambda}{z}-i p_{\perp} \cdot z_{\perp}}\left\langle 0\left|\left[ \pm \infty m+\infty z_{\perp} ; \pm \infty m\right][ \pm \infty m ; 0] q_{i}(0)\right| P_{h} S_{h} ; X\right\rangle \\
& \times\left\langle P_{h} S_{h} ; X\left|\bar{q}_{j}\left(\lambda m+z_{\perp}\right)\left[\lambda m+z_{\perp} ; \pm \infty m+z_{\perp}\right]\left[ \pm \infty m+z_{\perp} ; \pm \infty m+\infty z_{\perp}\right]\right| 0\right\rangle .
\end{aligned}
$$




\begin{tabular}{|c|c|c|c|}
\hline $\mathrm{N} \backslash \mathrm{q}$ & Ave. & $S_{\|}$ & $S_{\perp}$ \\
\hline Ave. & & & $h_{1}^{\perp(1)}(x)$ \\
\hline$S_{\|}$ & & & $h_{1 L}^{\perp(1)}(x)$ \\
\hline$S_{\perp}$ & $f_{1 T}^{\perp(1)}(x)$ & $g_{1 T}^{(1)}(x)$ & \\
\hline
\end{tabular}

\begin{tabular}{|c|c|c|c|}
\hline $\mathrm{h} \backslash \mathrm{q}$ & Ave. & $S_{\|}$ & $S_{\perp}$ \\
\hline Ave. & & & $H_{1}^{\perp(1)}(z)$ \\
\hline$S_{\|}$ & & & $H_{1 L}^{\perp(1)}(z)$ \\
\hline$S_{\perp}$ & $D_{1 T}^{\perp(1)}(z)$ & $G_{1 T}^{(1)}(z)$ & \\
\hline
\end{tabular}

Table 2: Kinematical twist-3 distribution functions (left) and fragmentation functions (right) classified by the spin of the hadron and a quark.

Decomposition of $\Phi_{\partial, i j}^{\rho}(x)$ and $\Delta_{\partial, i j}^{\rho}(z)$ defines kinematical twist-3 functions as listed in Table 2.

The third type of the twist- 3 functions are called dynamical twist-3 functions and defined from the quark-gluon correlator:

$$
\begin{aligned}
& \Phi_{F, i j}^{\rho}\left(x, x^{\prime}\right)= \int_{-\infty}^{\infty} \frac{d \lambda}{2 \pi} \int_{-\infty}^{\infty} \frac{d \mu}{2 \pi} \mathrm{e}^{i x^{\prime} \lambda+i\left(x-x^{\prime}\right) \mu}\left\langle P, S\left|\bar{q}_{j}(0)[0 ; \mu n] \operatorname{ign} F_{\eta} F^{\eta \rho}(\mu n)[\mu n ; \lambda n] q_{i}(\lambda n)\right| P, S\right\rangle, \\
& \Delta_{F, i j}^{\rho}\left(z, z^{\prime}\right)=\frac{1}{N_{c}} \sum_{X} \int_{-\infty}^{\infty} \frac{d \lambda}{2 \pi} \int_{-\infty}^{\infty} \frac{d \mu}{2 \pi} \mathrm{e}^{i \frac{\lambda}{z}+i\left(\frac{1}{z}-\frac{1}{z}\right) \mu} \\
& \quad \times\left\langle 0\left|[ \pm \infty m ; \mu m] \operatorname{igm}_{\eta} F^{\eta \rho}(\mu m)[\mu m ; \lambda m] q_{i}(\lambda m)\right| P_{h} S_{h} ; X\right\rangle \\
& \quad \times\left\langle P_{h} S_{h} ; X\left|\bar{q}_{j}(0)[0 ; \pm \infty m]\right| 0\right\rangle .
\end{aligned}
$$

Decomposition of $\Phi_{F, i j}^{\rho}\left(x, x^{\prime}\right)$ and $\Delta_{F, i j}^{\rho}\left(z, z^{\prime}\right)$, respectively, defines the dynamical twist-3 functions as listed in Table 3. The dynamical twist-3 distribution functions are real, and have definite symmetry under $x_{1} \leftrightarrow x_{2}: H_{F U}$ and $F_{F T}$ are symmetric, while $H_{F L}$ and $G_{F T}$ are anti-symmetric. The dynamical twist-3 fragmentation functions are complex functions: The real part is naively $T$-even, while imaginary part is naively $T$-odd. Neither real nor imaginary parts have definite symmetry properties. The support of dynamical twist- 3 functions is $-1<\left|x_{1,2}\right|<1,-1<\left|x_{2}-x_{1}\right|<1$ for distribution functions and $0<z_{1}<1, z_{1}<z_{2}<\infty$ for the fragmentation functions.

\begin{tabular}{|c|c|}
\hline Ave. & $H_{F U}\left(x_{1}, x_{2}\right)$ \\
\hline$S_{\|}$ & $H_{F L}\left(x_{1}, x_{2}\right)$ \\
\hline$S_{\perp}$ & $F_{F T}\left(x_{1}, x_{2}\right), G_{F T}\left(x_{1}, x_{2}\right)$ \\
\hline
\end{tabular}

\begin{tabular}{|c|c|}
\hline Ave. & $\widehat{H}_{F U}\left(z_{1}, z_{2}\right)$ \\
\hline$S_{\|}$ & $\widehat{H}_{F L}\left(z_{1}, z_{2}\right)$ \\
\hline$S_{\perp}$ & $\widehat{D}_{F T}\left(z_{1}, z_{2}\right), \widehat{G}_{F T}\left(z_{1}, z_{2}\right)$ \\
\hline
\end{tabular}

Table 3: Dynamical twist-3 distribution functions (left) and fragmentation functions (right) classified by the hadron spin configuration.

\section{Operator constraints for the twist-3 functions}

The twist-3 functions given in Tables 1-3 are not independent but have to obey constraint relations which follow from the QCD equation of motion (EOM) and the Lorentz invariance property of the correlator for each distribution and fragmentation function. The latter is a nonlocal version of the operator product expansion (OPE). In [1], we derived the complete set of those relations, in particular, those for the twist-3 fragmentation functions are new. Here we list the results. 


\subsection{EOM relations}

\subsubsection{EOM relations for the twist-3 distribution functions}

(i) Unpolarized nucleon:

$$
x e(x)=\frac{m_{q}}{M} f_{1}(x)-2 \mathscr{P} \int_{-1}^{1} d x^{\prime} \frac{H_{F U}\left(x, x^{\prime}\right)}{x-x^{\prime}},
$$

where $\mathscr{P}$ denotes the principal value prescription.

(ii)Transversely polarized nucleon:

$$
g_{1 T}^{(1)}(x)=x g_{T}(x)-\frac{m_{q}}{M} h_{1}(x)+\mathscr{P} \int_{-1}^{1} d x^{\prime} \frac{F_{F T}\left(x, x^{\prime}\right)-G_{F T}\left(x, x^{\prime}\right)}{x-x^{\prime}} .
$$

(iii) Longitudinally polarized nucleon:

$$
h_{1 L}^{\perp(1)}(x)=-\frac{x}{2} h_{L}(x)+\frac{m_{q}}{2 M} g_{1}(x)-\mathscr{P} \int_{-1}^{1} d x^{\prime} \frac{H_{F L}\left(x, x^{\prime}\right)}{x-x^{\prime}},
$$

\subsubsection{EOM relations for the twist-3 fragmentation functions}

(i) Unpolarized hadron:

$$
\begin{aligned}
& H_{1}^{\perp(1)}(z)=-\frac{H(z)}{2 z}+\int_{z}^{\infty} \frac{d z^{\prime}}{z^{\prime 2}} \frac{\mathfrak{I}\left[\hat{H}_{F U}\left(z, z^{\prime}\right)\right]}{\frac{1}{z}-\frac{1}{z^{\prime}}}, \\
& -\frac{E(z)}{2 z}=\int_{z}^{\infty} \frac{d z^{\prime}}{z^{\prime 2}} \frac{\Re\left[\hat{H}_{F U}\left(z, z^{\prime}\right)\right]}{\frac{1}{z}-\frac{1}{z^{\prime}}}-\frac{m_{q}}{2 M_{h}} D_{1}(z),
\end{aligned}
$$

where $\mathfrak{I}$ and $\mathfrak{R}$ represent imaginary and real parts, respectively.

(ii) Transversely polarized spin-1/2 hadron:

$$
\begin{aligned}
D_{1 T}^{\perp(1)}(z) & =-\frac{D_{T}(z)}{z}+\int_{z}^{\infty} \frac{d z^{\prime}}{z^{\prime 2}} \frac{\mathfrak{I}\left[\hat{D}_{F T}\left(z, z^{\prime}\right)\right]-\mathfrak{I}\left[\hat{G}_{F T}\left(z, z^{\prime}\right)\right]}{\frac{1}{z}-\frac{1}{z^{\prime}}}, \\
G_{1 T}^{(1)}(z) & =\frac{G_{T}(z)}{z}-\frac{m_{q}}{M_{h}} H_{1}(z)+\int_{z}^{\infty} \frac{d z^{\prime}}{z^{\prime 2}} \frac{\Re\left[\hat{D}_{F T}\left(z, z^{\prime}\right)\right]-\mathfrak{R}\left[\hat{G}_{F T}\left(z, z^{\prime}\right)\right]}{\frac{1}{z}-\frac{1}{z^{\prime}}} .
\end{aligned}
$$

(iii) Longitudinally polarized spin-1/2 hadron:

$$
\begin{aligned}
\frac{E_{L}(z)}{2 z} & =-\int_{z}^{\infty} \frac{d z^{\prime}}{z^{\prime 2}} \frac{\mathfrak{I}\left[\hat{H}_{F L}\left(z, z^{\prime}\right)\right]}{\frac{1}{z}-\frac{1}{z^{\prime}}} \\
H_{1 L}^{\perp(1)}(z) & =-\frac{H_{L}(z)}{2 z}+\frac{m_{q}}{2 M_{h}} G_{1}(z)-\int_{z}^{\infty} \frac{d z^{\prime}}{z^{\prime 2}} \frac{\Re\left[\hat{H}_{F L}\left(z, z^{\prime}\right)\right]}{\frac{1}{z}-\frac{1}{z^{\prime}}} .
\end{aligned}
$$

\subsection{Lorentz invariance relations}

In this subsection, we list the relations which follows from the Lorentz invariance of the correlators. We present the relations in the form of the so-called Lorentz invariance relations (LIR) which are obtained by combining the OPE relations with the EOM relations. 


\subsubsection{LIRs for twist-3 distribtuion functions}

(i) Transversely polarized nucleon:

$$
g_{T}(x)=g_{1}(x)+\frac{d}{d x} g_{1 T}^{(1)}(x)-2 \mathscr{P} \int_{-1}^{1} d x^{\prime} \frac{G_{F T}\left(x, x^{\prime}\right)}{\left(x-x^{\prime}\right)^{2}} .
$$

(ii) Longitudinally polarized nucleon:

$$
h_{L}(x)=h_{1}(x)-\frac{d}{d x} h_{1 L}^{\perp(1)}(x)+2 \mathscr{P} \int_{-1}^{1} d x^{\prime} \frac{H_{F L}\left(x, x^{\prime}\right)}{\left(x-x^{\prime}\right)^{2}} .
$$

\subsubsection{LIRs for twist-3 fragmentation functions}

(i) Transversely polarized spin-1/2 hadron:

$$
\begin{gathered}
\frac{G_{T}(z)}{z}=\frac{G_{1}(z)}{z}+\left(1-z \frac{d}{d z}\right) G_{1 T}^{(1)}(z)-\frac{2}{z} \int_{z}^{\infty} \frac{d z^{\prime}}{z^{\prime 2}} \frac{\Re\left[\hat{G}_{F T}\left(z, z^{\prime}\right)\right]}{\left(1 / z-1 / z^{\prime}\right)^{2}}, \\
\frac{D_{T}(z)}{z}=-\left(1-z \frac{d}{d z}\right) D_{1 T}^{\perp(1)}(z)-\frac{2}{z} \int_{z}^{\infty} \frac{d z^{\prime}}{z^{\prime 2}} \frac{\mathfrak{I}\left[\hat{D}_{F T}\left(z, z^{\prime}\right)\right]}{\left(1 / z-1 / z^{\prime}\right)^{2}} .
\end{gathered}
$$

(ii) Longitudinally polarized spin-1/2 hadron:

$$
\begin{gathered}
\frac{H_{L}(z)}{z}=\frac{H_{1}(z)}{z}-\left(1-z \frac{d}{d z}\right) H_{1 L}^{\perp(1)}(z)+\frac{2}{z} \int_{z}^{\infty} \frac{d z^{\prime}}{z^{\prime 2}} \frac{\mathfrak{R}\left[\hat{H}_{F L}\left(z, z^{\prime}\right)\right]}{\left(1 / z-1 / z^{\prime}\right)^{2}}, \\
\frac{H(z)}{z}=-\left(1-z \frac{d}{d z}\right) H_{1}^{\perp(1)}(z)-\frac{2}{z} \int_{z}^{\infty} \frac{d z^{\prime}}{z^{\prime 2}} \frac{\mathfrak{I}\left[\hat{H}_{F U}\left(z, z^{\prime}\right)\right]}{\left(1 / z-1 / z^{\prime}\right)^{2}} .
\end{gathered}
$$

Using the EOM relations and LIRs, one can eliminate the intrinsic and kinematical twist-3 functions in favor of the dynamical twist- 3 functions and the twist- 2 functions. In this sense the intrinsic and kinematical twist-3 functions may be regarded auxiliary functions. On the other hand, in the leading-order calculation, simple structure of partonic hard cross sections sometimes leads to a compact formula for the twist- 3 cross sections in terms of the intrinsic and kinematical twist- 3 functions $[2,3]$.

\section{Lorentz invariance of the twist- 3 cross sections}

Twist-3 cross sections can be obtained as a convolution of the hadronic correlators introduced in Sec. 1 and the corresponding hard part. The resulting twist-3 cross sections involve intrinsic, kinematical and dynamical twist-3 distribution and fragmentation functions together with twist-2 functions. In the decomposition of the hadronic correlators, the coefficients of the twist-3 functions contain lightlike vectors $n$ and $m$, and thus the expression for the cross sections also contain these vectors. On the other hand, the cross section should have a Lorentz invariant expression in terms of only the physical vectors such as momenta of the particles participating in the process and the spin vectors. Though $n$ and $m$ are uniquely determined from $P$ and $P_{h}$, their expression in terms of 
the physical vectors depends on a frame we choose, which makes the frame-independence of the cross section unclear.

In our recent paper [1] (see also [4]), we showed that the EOM relations and LIRs lead to frame-independent expression for the twist-3 cross sections, using, as an example, the inclusive hadron production in the lepton-nucleon collision, $e(l)+P(P) \rightarrow h\left(P_{h}\right)+X$. We define Mandelstam variables for this process as $S=(P+l)^{2}, T=\left(P-P_{h}\right)^{2}$ and $U=\left(l-P_{h}\right)^{2}$. In the cross section, $l, P$ and $P_{h}$ can be regarded as lightlike in the twist-3 accuracy, and therefore $P$ and $P_{h}$ can be identified as $p$ and $p_{h}$, respectively. To get a cross section in terms of the physical momenta, we note that $n^{\mu}$ and $m^{\mu}$ can be expanded in terms of $P_{h}^{\mu}, P^{\mu}, l^{\mu}$ and $\varepsilon^{\mu l P P_{h}}$. Taking into account the conditions, $n^{2}=m^{2}=0, P \cdot n=1$ and $P_{h} \cdot m=1$, one may write

$$
\begin{aligned}
& n^{\mu}=\chi P_{h}^{\mu}+\frac{2+\chi T}{S} l^{\mu}+\left[\frac{\chi U(2+\chi T)}{2 S}+\frac{S T U}{8} \chi_{\varepsilon}^{2}\right] P^{\mu}+\chi_{\varepsilon} \varepsilon^{\mu l P P_{h}}, \\
& m^{\mu}=\eta P^{\mu}-\frac{2+\eta T}{U} l^{\mu}+\left[\frac{\eta S(2+\eta T)}{2 U}+\frac{S T U}{8} \eta_{\varepsilon}^{2}\right] P_{h}^{\mu}+\eta_{\varepsilon} \varepsilon^{\mu l P P_{h}} .
\end{aligned}
$$

In the $e N$ center-of-mass frame, $n^{\mu}=2 l^{\mu} / S$, which corresponds to $\chi=\chi_{\varepsilon}=0$. In the $h P$ centerof-mass frame, $n^{\mu}=-2 P_{h}^{\mu} / T$ and $m^{\mu}=-2 P^{\mu} / T$, which corresponds to $\chi=\eta=-2 / T$ and $\chi_{\varepsilon}=\eta_{\varepsilon}=0$. After obtaining the twist- 3 cross sections in terms of $n$ and $m$, one can eliminate $n$ and $m$ by (3.1) and (3.2). For $e N^{\uparrow} \rightarrow h X, \vec{e} N^{\uparrow} \rightarrow h X, e N \rightarrow \Lambda^{\uparrow} X$ and $\vec{e} N \rightarrow \Lambda^{\uparrow} X$, we have shown that the EOM relations and LIRs, and

$$
\pi F_{F T}(x, x)=f_{1 T}^{\perp(1)}(x), \quad \pi H_{F U}(x, x)=h_{1}^{\perp(1)}(x),
$$

lead to Lorentz invariant expression for the twist-3 cross sections which are independent of the parameters $\chi, \chi_{\varepsilon}, \eta$ and $\eta_{\varepsilon}$. Therefore the frame-independence of the twist- 3 cross sections is guaranteed.

\section{Acknowledgments}

This work has been supported by the Grant-in-Aid for Scientific Research from the Japanese Society of Promotion of Science under Contract No. 26287040 (Y.K.), the National Science Foundation under Contract No. PHY-1516088 (K.K. and A.M.), the RIKEN BNL Research Center (D.P.), and the Bundesministerium für Bildung und Forschung (BMBF) grant 05P12VTCTG (MS).

\section{References}

[1] K. Kanazawa, Y. Koike, A. Metz, D. Pitonyak and M. Schlegel, Phys. Rev. D 93, no. 5, 054024 (2016) [arXiv:1512.07233 [hep-ph]].

[2] Y. Koike, D. Pitonyak, Y. Takagi and S. Yoshida, Phys. Lett. B 752, 95 (2016) [arXiv:1508.06499 [hep-ph]].

[3] Y. Koike, D. Pitonyak and S. Yoshida, Phys. Lett. B 759, 75 (2016) [arXiv:1603.07908 [hep-ph]].

[4] K. Kanazawa, A. Metz, D. Pitonyak and M. Schlegel, Phys. Lett. B 742, 340 (2015) [arXiv:1411.6459 [hep-ph]]. 